7. Sohn KM, Peck KR, Joo EJ, et al. Duration of colonization and risk factors for prolonged carriage of vancomycin-resistant enterococci after discharge from the hospital. Int $J$ Infect Dis 2013; 17:e240-e246.

8. Shenoy ES, Paras ML, Noubary F, Walensky RP, Hooper DC. Natural history of colonization with methicillin-resistant Staphylococcus aureus (MRSA) and vancomycin-resistant Enterococcus (VRE): a systematic review. BMC Infectious Dis 2014;14:177.

\section{Increase in Prevalence of KPC-2-Producing Klebsiella pneumoniae Recovered From Respiratory Secretions of Intensive Care Patients-Getting a Free Ride on a Menacing Colistin Resistance}

To the Editor-In recent years, carbapenem-resistant Enterobacteriaceae (CRE) have emerged as important nosocomial pathogens, while Klebsiella pneumoniae carbapenemase (KPC) has been the main carbapenem-resistant mechanism among CRE. ${ }^{1,2}$

Polymyxins (polymyxin B and colistin) have been widely applied because they are among the few agents that remain effective against multidrug-resistant Gram-negative bacteria such as carbapenem-resistant Pseudomonas aeruginosa, carbapenem-resistant Acinetobacter spp., and KPC producers. ${ }^{3}$ Although their clinical usefulness is increasing in most treatment strategies, the emergence of colistin resistance has been detected worldwide. ${ }^{4-6}$

To date, risk factors for KPC infection (eg, from prior intestinal colonization) have been extensively studied, but the definitive role of the presence of KPC producers from airways has remained uncertain. ${ }^{7}$ The widespread dissemination of colistin resistance among KPC producers has strong implications regarding colistin's utility, mainly in the setting of continuous selective pressure from the high-level use of the same, mostly in intensive care units. Therefore, a retrospective survey from January to June, 2016, including adult intensive care patients in a tertiary hospital in Porto Alegre, Southern Brazil, was conducted to assess the cumulative prevalence of KPC-producers, Acinetobacter spp., and Pseudomonas aeruginosa isolates recovered from endotracheal secretions of mechanically ventilated patients. Additionally, for these bacterial isolates, including $>1$ per patient from the same clinical site, susceptibility to colistin was evaluated to monitor how quickly colistin resistance could spread over the time.

Identification of bacterial species as well as carbapenem and colistin resistance was initially detected using an automated broth microdilution system (MicroScan; Beckman Coulter,
Brea, CA), followed by confirmation with Etest (AB Biodisk, Solna, Sweden). For enterobacterial species, a synergistic test was applied using phenyl-boronic acid to detect KPC and ethylenediaminetetraacetic acid to detect New Delhi metallobetalactamase, followed by gene detection by PCR analysis as described elsewhere. ${ }^{8}$

In total, 94 distinct patients were enrolled during the study period. Among the species considered in this study, only Acinetobacter baumannii, P. aeruginosa and KPC-2-producing Klebsiella pneumoniae (KPC-2-Kp) were detected. Cumulative prevalences for these isolates are shown in Figure 1A. Notably, the cumulative prevalences remained stable for A. baumannii $(27.9 \% \pm 2.74)$ and $P$. aeruginosa $(28.9 \% \pm 3.74)$ over the study period. No colistin resistance was observed over the follow-up period despite the high consumption of this class of drugs in this nosocomial setting over the same period of this survey (data not shown). In contrast, KPC-2-Kp $(14.3 \% \pm 5.27)$ showed a significant increase in prevalence during this period, ranging from $10.5 \%$ in January to $20.2 \%$ in June (odds ratio, 2.15; 95\% confidence interval, 0.4-10.1).

A total of $25 \mathrm{KPC}-2-\mathrm{Kp}$ isolates, recovered from 94 patients, were included over the study period: 4 isolates presenting no colistin resistant were recovered in the initial period (January) and 11 colistin-resistant isolates ( 11 of $25,44 \%$ ) were found in the final period (January to June). The linear trend shows that colistin resistance among $\mathrm{KPC}-2-K p$ is increasing drastically at a steady rate (Figure $1 \mathrm{~B}$ ).

In this study, endotracheal secretions presenting $\geq 10^{5} \mathrm{CFU} / \mathrm{mL}$ were selected to better reflect the influence of the use of colistin. Colistin is used to treat carbapenem-resistant $P$. aeruginosa and carbapenem-resistant A. baumannii, which contribute $\sim 33.5 \%$ and $97.5 \%$ of carbapenem resistance, respectively; these isolates were more prevalent from respiratory secretions of ICU patients during the same evaluated period. As previously reported by Lee et $\mathrm{al},{ }^{9}$ the emergence of colistin resistance among KPC-2-Kp isolates, which was also found in this study, may be explained by acquisition and development of resistance in the same isolate initially susceptible, or reinfection with a resistant isolate from a heterogeneous bacterial population under antibiotic selective pressure, as previously shown for fosfomycin ${ }^{10}$ or polymyxin $\mathrm{B}$ when rectal surveillance KPC-2-Kp isolates were evaluated. ${ }^{6}$ Although the impact of the presence of KPC on patient outcome has not been evaluated in this study, interest in the early diagnosis and prompt treatment of airway infections is growing, particularly concerning multidrug-resistant pathogens, such as $\mathrm{KPC}$-producers, in the effort to reduce rates of inappropriate empirical therapy. ${ }^{7}$

An ideal control is one that, in addition to preventing the spread of multidrug-resistant organisms in the environment, can also stagnate (and eliminate, if possible) its antimicrobial resistance levels. This is a fact. However, controlling these 2 fronts is not easy, and finding the balance between them to promote patient safety may be the greatest challenge of our time.

In conclusion, an increase in prevalence of KPC-2-Kp at a clinical site originally with low prevalence is reported in 
(A)

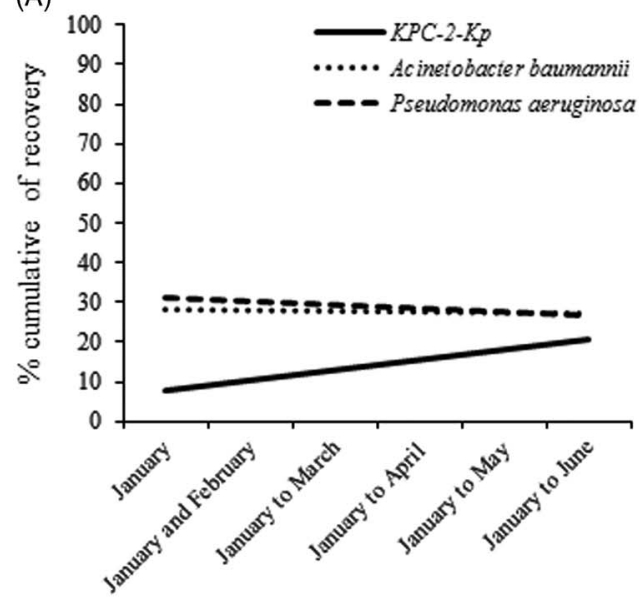

Cumulative period

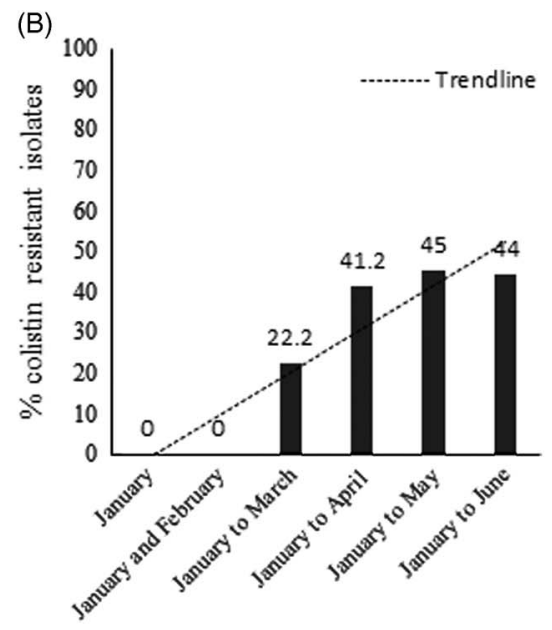

Cumulative period

FIGURE 1. Cumulative prevalence of KPC-2-Kp, A. baumannii and P. aeruginosa recovered from endotracheal secretions (A) and increase in colistin resistance among respiratory $\mathrm{KPC}-2-\mathrm{Kp}$ isolates (B) during the period study.

this study. Moreover, this increase seems to be supported by a high rate of colistin resistance among these KPC-2-Kp isolates, highlighting the urgent need for implementation of a strict stewardship program for prudent use of colistin to oppose this emerging resistance.

\section{A C KNOW LEDGMENTS}

Financial support. This work was supported in part by Conselho Nacional de Desenvolvimento Científico e Tecnológico (CNPq), Brazil.

Potential conflicts of interest. The author reports no conflicts of interest relevant to this article.

\section{Leandro Reus Rodrigues Perez, $\mathrm{PhD}^{1,2}$}

Affiliation: 1. Hospital Mãe de Deus, Porto Alegre, Brazil; 2. Universidade Federal do Rio Grande do Sul, Porto Alegre, Brazil.

Address correspondence to Leandro Reus Rodrigues Perez, PhD, Microbiology Unit - Hospital Mãe de Deus, 286, José de Alencar street, 90610-000 (leandro.reus@gmail.com).

Infect Control Hosp Epidemiol. 2016;37:1521-1522

(C) 2016 by The Society for Healthcare Epidemiology of America. All rights reserved. 0899-823X/2016/3712-0026. DOI: 10.1017/ice.2016.231

\section{REFERENCES}

1. van Duin D, Doi Y. Outbreak of colistin-resistant, carbapenemase-producing Klebsiella pneumoniae: Are we at the end of the road? J Clin Microbiol 2015;53:3116-3117.

2. Munoz-Price LS, Poirel L, Bonomo RA, et al. Clinical epidemiology of the global expansion of Klebsiella pneumoniae carbapenemases. Lancet Infect Dis 2013;13:785-796.
3. Lee GC, Burgess DS. Treatment of Klebsiella pneumoniae carbapenemase (KPC) infections: a review of published case series and case reports. Ann Clin Microbiol Antimicrob 2012; 11:32.

4. Giacobbe DR, Del Bono V, Trecarichi EM, et al. Risk factors for bloodstream infections due to colistin-resistant KPCproducing Klebsiella pneumoniae: results from a multicenter casecontrol-control study. Clin Microbiol Infect 2015;21:1106.e1-e8.

5. Giani T, Arena F, Vaggelli G, et al. Large nosocomial outbreak of colistin-resistant, carbapenemase-producing Klebsiella pneumoniae traced to clonal expansion of an $m g r \mathrm{~B}$ deletion mutant. J Clin Microbiol 2015;53:3341-3344.

6. Rodrigues Perez LR, Dias CG. Emergence of infections due to a polymyxin B-resistant KPC-2-producing Klebsiella pneumoniae in critically ill patients: What is the role of a previous colonization? Infect Control Hosp Epidemiol 2016;37:240-241.

7. Sbrana F, Malacarne P, Bassetti M, et al. Risk factors for ventilator associated pneumonia due to KPC-producing Klebsiella pneumoniae in mechanically ventilated patients with tracheal and rectal colonization. Minerva Anestesiol 2016;82:635-640.

8. Rodrigues Perez LR. Carbapenem-resistant Enterobacteriaceae: a major prevalence difference due to the high performance of carbapenemase producers when compared to the nonproducers. Infect Control Hosp Epidemiol 2015;36:1480-1482.

9. Lee J, Patel G, Huprikar S, Calfee DP, Jenkins SG. Decreased susceptibility to polymyxin B during treatment for carbapenemresistant Klebsiella pneumoniae infection. J Clin Microbiol 2009;47:1611-1612.

10. Perez LR. Menacing emergence of fosfomycin resistance among Klebsiella pneumoniae carbapenemase-2-producing K. pneumoniae driven by prior use in critically ill patients. Infect Control Hosp Epidemiol 2016;37:748-749. 